TRANSACTIONS OF THE

AMERICAN MATHEMATICAL SOCIETY

Volume 363, Number 2, February 2011, Pages 987-1005

S 0002-9947(2010)05136-0

Article electronically published on September 21, 2010

\title{
THE COMBINATORICS OF $k$-MARKED DURFEE SYMBOLS
}

\author{
KATHY QING JI
}

\begin{abstract}
George E. Andrews recently introduced $k$-marked Durfee symbols which are connected to moments of Dyson's rank. By these connections, Andrews deduced their generating functions and some combinatorial properties and left their purely combinatorial proofs as open problems. The primary goal of this article is to provide combinatorial proofs in answer to Andrews' request. We obtain a partition identity, which gives a relation between $k$-marked Durfee symbols and Durfee symbols by constructing bijections, and all identities on $k$-marked Durfee symbols given by Andrews could follow from this identity. In a similar manner, we also prove the identities due to Andrews on $k$-marked odd Durfee symbols combinatorially, which resemble ordinary $k$-marked Durfee symbols with a modified subscript and with odd numbers as entries.
\end{abstract}

\section{INTRODUCTION}

We will adopt the terminology on partitions in Andrews [2]. A partition $\lambda$ of a positive integer $n$ is a finite nonincreasing sequence of positive integers $\left(\lambda_{1}, \lambda_{2}, \ldots\right.$, $\left.\lambda_{r}\right)$ such that $\sum_{i=1}^{r} \lambda_{i}=n$. Then the $\lambda_{i}$ are called the parts of $\lambda$, where $\lambda_{1}$ is its largest part. The number of parts of $\lambda$ is called the length of $\lambda$, denoted by $l(\lambda)$. The weight of $\lambda$ is the sum of the parts of $\lambda$, denoted by $|\lambda|$.

The rank of a partition $\lambda$ introduced by Dyson [12 is defined as the largest part minus the number of parts, which is usually denoted by $r(\lambda)=\lambda_{1}-l(\lambda)$. Letting $N(m ; n)$ denote the number of partitions of $n$ with rank $m$, we have

Theorem 1.1 (Dyson). The generating function for $N(m ; n)$ is given by

$$
\sum_{n=0}^{+\infty} N(m ; n) q^{n}=\frac{1}{(q ; q)_{\infty}} \sum_{n=1}^{+\infty}(-1)^{n-1} q^{n(3 n-1) / 2+|m| n}\left(1-q^{n}\right), \quad|q|<1,
$$

where $(a ; q)_{n}=\prod_{j=0}^{n-1}\left(1-a q^{j}\right)$ and $(a ; q)_{\infty}=\lim _{n \rightarrow \infty}(a ; q)_{n}$.

The identity (1.1) was discovered by Dyson [12 in 1944 and proved by Atkin and Swinnerton-Dyer [5] in 1954. In 1969, Dyson [13] found a simple combinatorial argument of it. We refer to [7, p.63] for more details.

Definition 1.2. For a nonnegative integer $n$, a Durfee symbol of $n$ is a two-rowed array with a subscript

$$
(\alpha, \beta)_{D}=\left(\begin{array}{cccc}
\alpha_{1} & \alpha_{2} & \cdots & \alpha_{s} \\
\beta_{1} & \beta_{2} & \cdots & \beta_{t}
\end{array}\right)_{D},
$$

Received by the editors June 16, 2008 and, in revised form, June 8, 2009.

2010 Mathematics Subject Classification. Primary 11P81, 05A17, 05A19.

Key words and phrases. Rank, the moment of rank, the symmetrized moment of rank, Durfee symbols, $k$-marked Durfee symbols, odd Durfee symbols, $k$-marked odd Durfee symbols.

(C)2010 American Mathematical Society Reverts to public domain 28 years from publication 
where $D \geq \alpha_{1} \geq \alpha_{2} \geq \cdots \geq \alpha_{s}>0, D \geq \beta_{1} \geq \beta_{2} \geq \cdots \geq \beta_{t}>0$ and $n=\sum_{i=1}^{s} \alpha_{i}+\sum_{i=1}^{t} \beta_{i}+D^{2}$.

For example, there are 5 Durfee symbols of 4 :

$$
\left(\begin{array}{lll}
1 & 1 & 1
\end{array}\right)_{1}\left(\begin{array}{ll}
1 & 1 \\
1 &
\end{array}\right)_{1}\left(\begin{array}{ll}
1 & \\
1 & 1
\end{array}\right)_{1}\left(\begin{array}{lll}
1 & 1 & 1
\end{array}\right)_{1}\left(\begin{array}{l}
)_{2} \\
\end{array}\right.
$$

The difference between the lengths of $\alpha$ and $\beta$ is called the rank of Durfee symbols $(\alpha, \beta)_{D}$. We use $\mathcal{D}_{1}(m ; n)$ to denote the number of Durfee symbols of $n$ with rank $m$. Andrews [4, Section 3] showed the following theorem by constructing a bijection.

Theorem 1.3 (Andrews). The number of ordinary partitions of $n$ with rank $m$ is equal to the number of Durfee symbols of $n$ with rank $m$, that is,

$$
N(m ; n)=\mathcal{D}_{1}(m ; n) \text {. }
$$
by

$$
\eta_{k}(n)=\sum_{m=-\infty}^{+\infty}\left(\begin{array}{c}
m+\left\lfloor\frac{k-1}{2}\right\rfloor \\
k
\end{array}\right) N(m, n),
$$

which are linear combinations of the $k$ th rank moments $N_{k}(n)$,

$$
N_{k}(n)=\sum_{m=-\infty}^{+\infty} m^{k} N(m, n)
$$

considered by Atkin and Garvan [6]. To give a combinatorial explanation of (1.4), Andrews 4 introduced $k$-marked Durfee symbols, which can be thought of as the generalized Durfee symbols.

Definition 1.4. A $k$-marked Durfee symbol of $n$ is composed of $k$ pairs of partitions and a subscript, which is defined as

$$
\eta=\left(\begin{array}{cccc}
\alpha^{k}, & \alpha^{k-1}, & \ldots, & \alpha^{1} \\
\beta^{k}, & \beta^{k-1}, & \ldots, & \beta^{1}
\end{array}\right)_{D},
$$

where $\alpha^{i}$ (resp. $\beta^{i}$ ) represents a partition and $\sum_{i=1}^{k}\left(\left|\alpha^{i}\right|+\left|\beta^{i}\right|\right)+D^{2}=n$. Furthermore, the partitions $\alpha^{i}$ and $\beta^{i}$ must satisfy the following three conditions, where $\alpha_{1}^{i}$ (resp. $\beta_{1}^{i}$ ) is the largest part of the partition $\alpha^{i}$ (resp. $\left.\beta^{i}\right)$ and $\alpha_{l\left(\alpha^{i}\right)}^{i}$ (resp. $\left.\beta_{l\left(\beta^{i}\right)}^{i}\right)$ is the smallest part of the partition $\alpha^{i}$ (resp. $\beta^{i}$ ).

(1) For $1 \leq i<k, \alpha^{i}$ must be a nonempty partition, while $\alpha^{k}$ and $\beta^{i}$ could be empty;

(2) $\beta_{1}^{i-1} \leq \alpha_{1}^{i-1} \leq \beta_{l\left(\beta^{i}\right)}^{i}$ for $2 \leq i \leq k$;

(3) $\beta_{1}^{k}, \alpha_{1}^{k} \leq D$.

Clearly, a 1-marked Durfee symbol is just a Durfee symbol.

Let $\eta=\left(\begin{array}{cccc}\alpha^{k}, & \alpha^{k-1}, & \ldots, & \alpha^{1} \\ \beta^{k}, & \beta^{k-1}, & \ldots, & \beta^{1}\end{array}\right)$ be a $k$-marked Durfee symbol. The pair of partitions $\left(\begin{array}{c}\alpha^{i} \\ \beta^{i}\end{array}\right)$ is called the $i$ th vector of $\eta$. We define $\rho_{i}(\eta)$, the $i$ th rank of $\eta$, by

$$
\rho_{i}(\eta)= \begin{cases}l\left(\alpha^{i}\right)-l\left(\beta^{i}\right)-1 & \text { for } 1 \leq i<k \\ l\left(\alpha^{k}\right)-l\left(\beta^{k}\right) & \text { for } i=k .\end{cases}
$$


For example, $\left(\begin{array}{llllll}4_{3} & 4_{3} & 3_{2} & 3_{2} & 2_{2} & 2_{1} \\ & 5_{3} & & 3_{2} & 2_{2} & 2_{1}\end{array}\right)_{5}$ is a 3-marked Durfee symbol of 55 where $\alpha^{3}=(4,4), \alpha^{2}=(3,3,2), \alpha^{1}=(2)$, and $\beta^{3}=(5), \beta^{2}=(3,2), \beta^{1}=(2)$. The first rank is -1 , the second rank is 0 , and the third rank is 1 .

Let $\mathcal{D}_{k}\left(m_{1}, m_{2}, \ldots, m_{k} ; n\right)$ denote the number of $k$-marked Durfee symbols of $n$ with $i$ th rank equal to $m_{i}$ and $\mathcal{D}_{k}(n)$ denote the number of $k$-marked Durfee symbols of $n$; it's clear to see that

$$
\mathcal{D}_{k}(n)=\sum_{m_{1}, \ldots, m_{k}=-\infty}^{+\infty} \mathcal{D}_{k}\left(m_{1}, m_{2}, \ldots, m_{k} ; n\right) .
$$

In the recent work 4, Andrews used the connections between $k$-marked Durfee symbols and the symmetrized rank moments (1.4) to find some identities relating the generating function for $\mathcal{D}_{k}\left(m_{1}, m_{2}, \ldots, m_{k} ; n\right)$, as well as to deduce some combinatorial properties and Ramanujan-type congruences for $k$-marked Durfee symbols. At the end of the paper, Andrews proposed a variety of serious questions which fall into 3 basic groups: combinatorial, asymptotic and congruential. The recent works 9. 10, by Kathrin Bringmann, Frank Garvan, and Karl Mahlburg focused on the study relating asymptotical and congruential properties of $k$-marked Durfee symbols. They used the automorphic properties to prove the existence of infinitely many congruences for $k$-marked Durfee symbols. Also, William J. Keith did some work on the congruential properties of $k$-marked Durfee symbols in [15, 16]. He mainly showed that $k$-marked Durfee symbols of $n$ equally populate the residue classes $a$ and $b \quad(\bmod 2 k+1)$ if $\operatorname{gcd}(a, 2 k+1)=\operatorname{gcd}(b, 2 k+1)$. The primary goal of this article is to answer Andrews' request on combinatorics (Problems 1-4 and 6-9 on page 39 of [4). We will give combinatorial proofs of the identities relating the generating function for $\mathcal{D}_{k}\left(m_{1}, m_{2}, \ldots, m_{k} ; n\right)$ and combinatorial properties for $k$-marked Durfee symbols. To be specific, we first derive the following partition identity (1.7) by constructing bijections, which gives a relation between $k$-marked Durfee symbols and Durfee symbols. We then show that all identities on $k$-marked Durfee symbols given by Andrews (4, Problems 1-4]) could follow from this identity. Finally, we use a similar method to study the identities of Andrews on $k$-marked odd Durfee symbols (4 4 , Problems 6-9]), which resemble ordinary Durfee symbols with a modified subscript and with odd numbers as entries.

Theorem 1.5. For $k \geq 2$, we have

$$
\mathcal{D}_{k}\left(m_{1}, m_{2}, \ldots, m_{k} ; n\right)=\sum_{j=0}^{+\infty}\left(\begin{array}{c}
j+k-2 \\
k-2
\end{array}\right) N\left(\sum_{i=1}^{k}\left|m_{i}\right|+2 j+k-1 ; n\right) .
$$

The referee has kindly pointed out that Kagan Kursungoz has also done some studies on the combinatorics of $k$-marked Durfee symbols in his doctorial thesis [17] and in a paper joint with Boulet 8. An alternative definition of $k$-marked Durfee symbols is given in 17. Using this alternative characterization, Kursungoz gave alternative proofs of Corollaries 12, 13 and Theorem 7 in [4] (that is, Problems 2-4 in [4), but his method could not be applied to prove Corollary 11 in [4] (that is, Problem 1 in [4). Moreover, his method is essentially different from this paper.

We now briefly describe the organization of this paper. In Section 2, we consider the relation between $k$-marked Durfee symbols and Durfee symbols and prove Theorem 1.5. To this end, we introduce a special class of $k$-marked Durfee symbols, 
which we call $k$-marked strict shifted Durfee symbols since each of their vectors except for the $k$ th vector is a two-lined strict shifted plane partition. We deduce the desired relation by building the connections between $k$-marked strict shifted Durfee symbols, Durfee symbols, and $k$-marked Durfee symbols, respectively. In Section 3, we give combinatorial proofs of the identities due to Andrews on $k$-marked Durfee symbols with the help of Theorem 1.5. In particular, the symmetry of $k$-marked Durfee symbols (4, Corollary 12]) could be thought of as a direct consequence of Theorem 1.5. Section 4 is devoted to the study of $k$-marked odd Durfee symbols.

\section{2. $k$-MARKED STRICT SHIFTED DURFEE SYMBOLS}

In this section, we will establish the relations between $k$-marked strict shifted Durfee symbols, Durfee symbols, and $k$-marked Durfee symbols, respectively, and then deduce Theorem 1.5. We begin by defining $k$-marked strict shifted Durfee symbols.

To define this object, we first need to give the definition of two-lined strict shifted plane partitions, which is a special case of strict shifted plane partitions introduced by Andrews 3. A two-lined strict shifted plane partition of $n$ is just a pair of partitions $(\alpha, \beta)$ of $n$, where $\alpha=\left(\alpha_{1}, \alpha_{2}, \ldots, \alpha_{r}\right), \beta=\left(\beta_{1}, \beta_{2}, \ldots, \beta_{s}\right), r>s$, and $\alpha_{i+1}>\beta_{i}$ for $i=1,2, \ldots, s$. For example $\left(\begin{array}{cccccc}3 & 3 & 3 & 2 & 2 & 1 \\ & 2 & 1 & 1 & 1 & \end{array}\right)$ is a two-lined strict shifted plane partition.

A $k$-marked Durfee symbol $\eta=\left(\begin{array}{cccc}\alpha^{k}, & \alpha^{k-1}, & \ldots, & \alpha^{1} \\ \beta^{k}, & \beta^{k-1}, & \ldots, & \beta^{1}\end{array}\right)_{D}$ is said to be strict shifted if all of its vectors except for the $k$ th vector $\left(\alpha^{k}, \beta^{k}\right)$ are two-lined strict shifted plane partitions. For example, the 3-marked Durfee symbol in (2.1) is strict shifted. Let $\mathcal{D}_{k}^{s s}\left(m_{1}, m_{2}, \ldots, m_{k} ; n\right)$ denote the number of $k$-marked strict shifted Durfee symbols of $n$ with $i$ th rank equal to $m_{i}$.

We now build a connection between $k$-marked strict shifted Durfee symbols of $n$ and Durfee symbols of $n$.

Theorem 2.1. Given $k$ nonnegative integers $m_{1}, m_{2}, \ldots, m_{k}$, there is a bijection $\Phi$ between the set of $k$-marked strict shifted Durfee symbols of $n$ with ith rank equal to $m_{i}$ and the set of Durfee symbols of $n$ with rank equal to $\sum_{i=1}^{k} m_{i}+k-1$.

Proof. The map $\Phi$ : Letting $\eta=\left(\begin{array}{cccc}\alpha^{k}, & \alpha^{k-1}, & \ldots, & \alpha^{1} \\ \beta^{k}, & \beta^{k-1}, & \ldots, & \beta^{1}\end{array}\right)$ be counted by $\mathcal{D}_{k}^{s s}\left(m_{1}, \ldots, m_{k} ; n\right)$, we now construct a Durfee symbol $(\gamma, \delta)_{D^{\prime}}$. First set $D^{\prime}=D$ and let $\gamma$ be the partition composed of all parts of $\alpha^{1}, \alpha^{2}, \ldots, \alpha^{k}$ and $\delta$ be the partition composed of all parts of $\beta^{1}, \beta^{2}, \ldots, \beta^{k}$. Obviously, the resulting Durfee symbol $(\gamma, \delta)_{D}$ is enumerated by $\mathcal{D}_{1}\left(\sum_{i=1}^{k} m_{i}+k-1 ; n\right)$.

The inverse map $\Phi^{-1}$ : Letting $(\gamma, \delta)_{D}$ be counted by $\mathcal{D}_{1}\left(\sum_{i=1}^{k} m_{i}+k-1 ; n\right)$, we will construct a $k$-marked strict shifted Durfee symbol

$$
\eta=\left(\begin{array}{cccc}
\alpha^{k}, & \alpha^{k-1}, & \ldots, & \alpha^{1} \\
\beta^{k}, & \beta^{k-1}, & \ldots, & \beta^{1}
\end{array}\right)_{D^{\prime}}
$$

whose $i$ th rank is equal to $m_{i}$. Letting

$$
\left(\begin{array}{l}
\gamma \\
\delta
\end{array}\right)_{D}=\left(\begin{array}{llll}
\gamma_{1} & \gamma_{2} & \ldots & \gamma_{l} \\
\delta_{1} & \delta_{2} & \ldots & \delta_{s}
\end{array}\right)_{D},
$$


where $D \geq \gamma_{1} \geq \gamma_{2} \geq \cdots \geq \gamma_{l}$ and $D \geq \delta_{1} \geq \delta_{2} \geq \cdots \geq \delta_{s}$, we assume that $\delta_{j}=0$ for $j \geq s+1$. Note that $l-s=\sum_{i=1}^{k} m_{i}+k-1$.

We now split $(\gamma, \delta)$ to generate the $k$ th vector $\left(\alpha^{k}, \beta^{k}\right)$ of $\eta$. Let $j$ be the largest nonnegative integer such that $\delta_{j} \geq \gamma_{m_{k}+j+1}$; that is, for any $i \geq j+1$, we have $\delta_{i}<\gamma_{m_{k}+i+1}$. Let

$$
\left(\begin{array}{c}
\alpha^{k} \\
\beta^{k}
\end{array}\right)=\left(\begin{array}{cccc}
\gamma_{1} & \gamma_{2} & \ldots & \gamma_{m_{k}+j} \\
\delta_{1} & \delta_{2} & \ldots & \delta_{j}
\end{array}\right)
$$

and

$$
\left(\begin{array}{c}
\gamma^{\prime} \\
\delta^{\prime}
\end{array}\right)=\left(\begin{array}{cccc}
\gamma_{1}^{\prime} & \gamma_{2}^{\prime} & \ldots & \gamma_{l^{\prime}}^{\prime} \\
\delta_{1}^{\prime} & \delta_{2}^{\prime} & \ldots & \delta_{s^{\prime}}^{\prime}
\end{array}\right)
$$

where $\gamma_{i}^{\prime}=\gamma_{m_{k}+j+i}, \delta_{i}^{\prime}=\delta_{j+i}$ for $i \geq 1$. Obviously, $l\left(\alpha^{k}\right)-l\left(\beta^{k}\right)=m_{k}$. Furthermore, $\left(\gamma^{\prime}, \delta^{\prime}\right)$ is a two-lined strict shifted plane partition from the fact that for any $i \geq 1, \delta_{i+j}<\gamma_{m_{k}+i+j+1}$ and $l^{\prime}-s^{\prime}=\sum_{i=1}^{k-1} m_{i}+k-1$.

We continue to split $\left(\gamma^{\prime}, \delta^{\prime}\right)$ to construct the $(k-1)$ th vector $\left(\alpha^{k-1}, \beta^{k-1}\right)$ of $\eta$. Letting $j$ be the largest nonnegative integer such that $\delta_{j}^{\prime} \geq \gamma_{m_{k-1}+j+2}^{\prime}$, we then let

$$
\left(\begin{array}{c}
\alpha^{k-1} \\
\beta^{k-1}
\end{array}\right)=\left(\begin{array}{cccc}
\gamma_{1}^{\prime} & \gamma_{2}^{\prime} & \ldots & \gamma_{m_{k-1}+j+1}^{\prime} \\
\delta_{1}^{\prime} & \delta_{2}^{\prime} & \ldots & \delta_{j}^{\prime}
\end{array}\right)
$$

and

$$
\left(\begin{array}{l}
\gamma^{\prime \prime} \\
\delta^{\prime \prime}
\end{array}\right)=\left(\begin{array}{cccc}
\gamma_{1}^{\prime \prime} & \gamma_{2}^{\prime \prime} & \ldots & \gamma_{l^{\prime \prime}}^{\prime \prime} \\
\delta_{1}^{\prime \prime} & \delta_{2}^{\prime \prime} & \ldots & \delta_{s^{\prime \prime}}^{\prime \prime}
\end{array}\right)
$$

where $\gamma_{i}^{\prime \prime}=\gamma_{m_{k-1}+j+i+1}^{\prime}, \delta_{i}^{\prime \prime}=\delta_{j+i}^{\prime}$ for $i \geq 1$. Clearly, $l\left(\alpha^{k-1}\right)-l\left(\beta^{k-1}\right)=m_{k-1}+1$ and $\left(\alpha^{k-1}, \beta^{k-1}\right)$ is a two-lined strict shifted plane partition since $\left(\gamma^{\prime}, \delta^{\prime}\right)$ is strict shifted. Observe that $\delta_{j+i}^{\prime}<\gamma_{m_{k-1}+i+j+2}^{\prime}$ for $i \geq 1$, so $\left(\gamma^{\prime \prime}, \delta^{\prime \prime}\right)$ is also strict shifted and $l^{\prime \prime}-s^{\prime \prime}=\sum_{i=1}^{k-2} m_{i}+k-2$.

Repeating the above process to generate $\left(\alpha^{k-2}, \beta^{k-2}\right), \ldots,\left(\alpha^{1}, \beta^{1}\right)$, respectively, and letting $D^{\prime}=D$, we obtain a $k$-marked strict shifted Durfee symbol

$$
\eta=\left(\begin{array}{cccc}
\alpha^{k}, & \alpha^{k-1}, & \ldots, & \alpha^{1} \\
\beta^{k}, & \beta^{k-1}, & \ldots, & \beta^{1}
\end{array}\right)_{D}
$$

which is counted by $\mathcal{D}_{k}^{s s}\left(m_{1}, m_{2}, \ldots, m_{k} ; n\right)$.

We now illustrate the inverse map $\Phi^{-1}$ by going through an example in details. Taking $m_{1}=1, m_{2}=1, m_{3}=0$, and letting

$$
\left(\begin{array}{l}
\gamma \\
\delta
\end{array}\right)_{D}=\left(\begin{array}{lllllllllll}
6 & 6 & 3 & 3 & 3 & 3 & 2 & 2 & 1 & 1 & 1 \\
5 & 5 & 4 & 2 & 1 & 1 & 1 & & & &
\end{array}\right)_{6},
$$

we first split $(\gamma, \delta)$ to get $\left(\alpha^{3}, \beta^{3}\right)$ and $\left(\gamma^{\prime}, \delta^{\prime}\right)$. Noting that the divisional part $\delta_{j}$ is the smallest part in $\delta$ satisfying $\delta_{j} \geq \gamma_{j+1}$,

$$
\left(\begin{array}{l}
\alpha^{3} \\
\beta^{3}
\end{array}\right)=\left(\begin{array}{lll}
6 & 6 & 3 \\
5 & 5 & 4
\end{array}\right),\left(\begin{array}{l}
\gamma^{\prime} \\
\delta^{\prime}
\end{array}\right)=\left(\begin{array}{llllllll}
3 & 3 & 3 & 2 & 2 & 1 & 1 & 1 \\
& 2 & 1 & 1 & 1 & & &
\end{array}\right),
$$

we then split $\left(\gamma^{\prime}, \delta^{\prime}\right)$ to generate $\left(\alpha^{2}, \beta^{2}\right)$ and $\left(\alpha^{1}, \beta^{1}\right)$, where the divisional part $\delta_{j}^{\prime}$ is the smallest part in $\delta^{\prime}$ satisfying $\delta_{j}^{\prime} \geq \gamma_{j+3}^{\prime}$,

$$
\left(\begin{array}{l}
\alpha^{2} \\
\beta^{2}
\end{array}\right)=\left(\begin{array}{llllll}
3 & 3 & 3 & 2 & 2 & 1 \\
2 & 1 & 1 & 1 &
\end{array}\right),\left(\begin{array}{l}
\alpha^{1} \\
\beta^{1}
\end{array}\right)=\left(\begin{array}{ll}
1 & 1 \\
&
\end{array}\right) .
$$


Thus we get

$$
\eta=\left(\begin{array}{lllllllllll}
6_{3} & 6_{3} & 3_{3} & 3_{2} & 3_{2} & 3_{2} & 2_{2} & 2_{2} & 1_{2} & 1_{1} & 1_{1} \\
5_{3} & 5_{3} & 4_{3} & & 2_{2} & 1_{2} & 1_{2} & 1_{2} & & &
\end{array}\right)_{6}
$$

By Theorems 2.1 and 1.3, we deduce the following partition identity.

Corollary 2.2. For $m_{i} \geq 0$ and $k \geq 1$, we have

$$
\mathcal{D}_{k}^{s s}\left(m_{1}, m_{2}, \ldots, m_{k} ; n\right)=N\left(\sum_{i=1}^{k} m_{i}+k-1 ; n\right) .
$$

To establish the relation between $k$-marked strict shifted Durfee symbols and $k$ marked Durfee symbols, we need to define a statistic on $k$-marked Durfee symbols. In the same way, we first define this statistic on a pair of partitions.

For $(\gamma, \delta)$, the part $\delta_{i}$ is said to be balanced if $\gamma_{i+1} \leq \delta_{i}$ and the number of parts greater than $\delta_{i}$ in $\gamma$ ( $\gamma_{1}$ is not counted) is equal to the number of unbalanced parts before $\delta_{i}$ in $\delta$. For example, let $\left(\begin{array}{c}\gamma \\ \delta\end{array}\right)=\left(\begin{array}{ccccc}4 & 3 & 3 & 1 & 1 \\ & \underline{3} & 2 & 2 & \end{array}\right)$, where the first part 3 of $\delta$ is balanced while the third part 2 is not balanced, although it satisfies the first condition $\gamma_{i+1} \leq \delta_{i}$. Because that there are two parts greater than 2 (4 is not counted) in $\gamma$, while there is only one unbalanced part (the second part 2) before the third part 2 in $\delta$.

It should be pointed out that for any an unbalanced part $\delta_{i}$ of $(\gamma, \delta)$, the number of parts greater than $\delta_{i}$ in $\gamma\left(\gamma_{1}\right.$ is not counted) is greater than the number of unbalanced parts before $\delta_{i}$ in $\delta$. We will state this in the following proposition:

Proposition 2.3. Let $(\gamma, \delta)$ be a pair of partitions. For any part $\delta_{j}$ of $\delta$, let $d_{j}$ denote the difference between the number of parts greater than $\delta_{j}$ in $\gamma$ (except for $\left.\gamma_{1}\right)$ and the number of unbalanced parts before $\delta_{j}$ in $\delta$. We then have $d_{j} \geq 0$.

For the above example, we see that $d_{1}=0, d_{2}=2, d_{3}=1$.

Proof. If $\gamma_{j+1}>\delta_{j}$, it's clear to see that $d_{j} \geq 1$; if $\gamma_{j+1} \leq \delta_{j}$, we consider the following two cases:

Case 1. When $\delta_{j-1}<\gamma_{j}$, and at this time, there are $j-1$ parts greater than $\delta_{j}$ in $\gamma\left(\gamma_{1}\right.$ is not counted) and the number of unbalanced parts before $\delta_{j}$ in $\delta$ is less than or equal to $j-1$, so $d_{j} \geq 0$.

Case 2. When $\delta_{j-1} \geq \gamma_{j}$, let $t$ be the largest nonnegative integer less than $j$ such that $\delta_{t}<\gamma_{t+1}$. Here we assume that $\delta_{0}=0$ and then $t$ must exist. From Case 1, we know that $d_{t+1} \geq 0$. We use induction to prove that $d_{i} \geq 0$ for $t+2 \leq i \leq j$.

We first prove that $d_{t+2} \geq 0$. Note that $\gamma_{t+1}>\delta_{t+1} \geq \gamma_{t+2}$. If $\delta_{t+2}<\gamma_{t+2}$, then $d_{t+2} \geq d_{t+1} \geq 0$; If $\delta_{t+2} \geq \gamma_{t+2}$, then $d_{t+2} \geq d_{t+1}-1$. In particular, if $d_{t+1}=0$, then $\delta_{t+1}$ is balanced; thus $d_{t+2}=d_{t+1}=0$. So $d_{t+2} \geq 0$.

Assume that $d_{i} \geq 0$ and $\gamma_{p}>\delta_{i} \geq \gamma_{p+1}$. We will prove that $d_{i+1} \geq 0$. If $\delta_{i+1} \geq \gamma_{p+1}$, a similar argument on the case for $\delta_{t+2}$ could show that $d_{i+1} \geq 0$. If $\delta_{i+1}<\gamma_{p+1}$, then $d_{i+1} \geq d_{i} \geq 0$.

We use $b(\gamma, \delta)$ to denote the number of balanced parts in $(\gamma, \delta)$. Clearly, $0 \leq$ $b(\gamma, \delta) \leq l(\delta)$. Letting $\eta=\left(\begin{array}{cccc}\alpha^{k}, & \alpha^{k-1}, & \ldots, & \alpha^{1} \\ \beta^{k}, & \beta^{k-1}, & \ldots, & \beta^{1}\end{array}\right)_{D}$ be a $k$-marked Durfee 
symbol, we define $\operatorname{nb}_{i}(\eta)$, called the $i$ th balanced number, by

$$
\operatorname{nb}_{i}(\eta)= \begin{cases}b\left(\alpha^{i}, \beta^{i}\right) & \text { for } 1 \leq i<k, \\ 0 & \text { for } i=k .\end{cases}
$$

For $\eta=\left(\begin{array}{llllllll}4_{3} & 4_{3} & 3_{2} & 2_{2} & 2_{2} & 2_{1} & 2_{1} & 1_{1} \\ 5_{3} & & & \underline{3_{2}} & \underline{2_{2}} & & \underline{2_{1}} & 1_{1}\end{array}\right)$, we have $\operatorname{nb}_{1}(\eta)=1, \operatorname{nb}_{2}(\eta)=$ $2, \operatorname{nb}_{3}(\eta)=0$.

We next state a theorem concerning two-lined strict shifted plane partitions.

Theorem 2.4. Given two nonnegative integers $r, m$, there is a bijection $\psi$ between the set of pairs of partitions $(\alpha, \beta)$ of $n$ with $\beta_{1} \leq \alpha_{1}$ where there are $r$ balanced parts and the difference between the lengths of $\alpha$ and $\beta$ is equal to $m$ and the set of two-lined strict shifted plane partitions $(\bar{\alpha}, \bar{\beta})$ of $n$ where the difference between the lengths of $\bar{\alpha}$ and $\bar{\beta}$ is equal to $m+2 r$.

Proof. The map $\psi$ : Let $(\alpha, \beta)$ be a pair of partitions with $r$ balanced parts and $l(\alpha)-l(\beta)=m$. The two-lined strict shifted plane partition $(\bar{\alpha}, \bar{\beta})$ is constructed as follows: $\bar{\alpha}$ is composed of all parts of $\alpha$ and all balanced parts of $\beta$. $\bar{\beta}$ consists of all unbalanced parts of $\beta$. As an example, let $\left(\begin{array}{c}\alpha \\ \beta\end{array}\right)=\left(\begin{array}{cccccc}6 & 5 & 5 & 3 & 3 & 2 \\ & \underline{5} & 4 & 4 & \underline{3} & \end{array}\right)$, where the underlined parts in $\beta$ are balanced. According to the above construction, we get $\left(\begin{array}{c}\bar{\alpha} \\ \bar{\beta}\end{array}\right)=\left(\begin{array}{cccccccc}6 & \underline{5} & 5 & 5 & \underline{3} & 3 & 3 & 2 \\ & 4 & 4 & & & & & \end{array}\right)$. It's clear to see that $|\alpha|+|\beta|=$ $|\bar{\alpha}|+|\bar{\beta}|, l(\bar{\alpha})-l(\bar{\beta})=l(\alpha)+r-(l(\beta)-r)=m+2 r$. By Proposition 2.3, one can easily know that $(\bar{\alpha}, \bar{\beta})$ is strict shifted.

The inverse map $\psi^{-1}$ : Let $(\bar{\alpha}, \bar{\beta})$ be a two-lined strict shifted plane partition, where the difference between the lengths of $\bar{\alpha}$ and $\bar{\beta}$ is $m+2 r$, that is, $l(\bar{\alpha})-l(\bar{\beta})=$ $m+2 r$. We now construct a pair of partitions $(\alpha, \beta)$ with $r$ balanced parts and $l(\alpha)-l(\beta)=m$.

First of all, attach a subscript $g_{i}$ for each part $\bar{\alpha}_{i}$ of $\bar{\alpha}$, where $g_{i}$ denotes the difference between the number of parts before $\bar{\alpha}_{i}$ in $\bar{\alpha}\left(\bar{\alpha}_{1}\right.$ is not counted) and the number of parts greater than or equal to $\bar{\alpha}_{i}$ in $\bar{\beta}$. We let $g_{1}=0$.

For example, if $\left(\begin{array}{c}\bar{\alpha} \\ \bar{\beta}\end{array}\right)=\left(\begin{array}{ccccccccc}6 & 5 & 5 & 5 & 3 & 3 & 3 & 2 & 1 \\ & 4 & 4 & 3 & & & & & \end{array}\right)$, attach the subscripts for all parts of $\bar{\alpha}$ to get $\left(\begin{array}{ccccccccc}6_{0} & 5_{0} & 5_{1} & 5_{2} & 3_{0} & 3_{1} & 3_{2} & 2_{3} & 1_{4} \\ & 4 & 4 & 3 & & & & & \end{array}\right)$.

One could easily know that $g_{2}=0$ and $g_{i} \geq 0$ for any $3 \leq i \leq l(\bar{\alpha})$ from the fact that $(\bar{\alpha}, \bar{\beta})$ is strict shifted. Letting $\bar{\alpha}_{t_{i}}$ be the smallest part in all parts of $\bar{\alpha}$ with subscript $i$, we have the following conclusion:

Lemma 2.5. For $0 \leq i \leq m+2 r-2$, $\bar{\alpha}_{t_{i}}$ exists, and $\bar{\alpha}_{t_{0}} \geq \bar{\alpha}_{t_{1}} \geq \cdots \geq \bar{\alpha}_{t_{m+2 r-2}}$.

In the above example, $m+2 r=6$ and $\bar{\alpha}_{t_{0}}=3, \bar{\alpha}_{t_{1}}=3, \bar{\alpha}_{t_{2}}=3, \bar{\alpha}_{t_{3}}=2, \bar{\alpha}_{t_{4}}=1$.

Proof. We use induction to show that the sequence of subscripts $\left\{g_{1}, g_{2}, \ldots, g_{l(\bar{\alpha})}\right\}$ consists of all nonnegative integers less than $m+2 r-1$. Obviously, 0 is in this sequence. Assume that $i$ is in this sequence, that is, there is a part $\bar{\alpha}_{p}$ such that $g_{p}=i$. We now prove that $i+1$ is also in this sequence. By the induction hypothesis, we know that the subscript of the part $\bar{\alpha}_{p}$ is $i$, that is, $\bar{\beta}_{p-i-1}<\bar{\alpha}_{p} \leq \bar{\beta}_{p-i-2}$. Letting $l(\bar{\beta})=s$ and noting that $i \leq m+2 r-3$, we have $s+i+3 \leq s+m+2 r=l(\bar{\alpha})$. If $\bar{\alpha}_{s+i+3} \leq \bar{\beta}_{s}$, then the subscript of $\bar{\alpha}_{s+i+3}$ is $i+1$. Otherwise, there must exist 
$p+1 \leq j \leq s+i+2$ such that $\bar{\beta}_{j-i-2}<\bar{\alpha}_{j} \leq \bar{\beta}_{j-i-3}$. This is because $\bar{\alpha}_{p+1} \leq \bar{\beta}_{p-i-2}$ and $\bar{\alpha}_{s+i+2}>\bar{\beta}_{s}$. Hence, the subscript of $\bar{\alpha}_{j}$ is $i+1$. Therefore, $\bar{\alpha}_{t_{i}}$ exists for $0 \leq i \leq m+2 r-2$ and $\bar{\alpha}_{t_{0}} \geq \bar{\alpha}_{t_{1}} \geq \cdots \geq \bar{\alpha}_{t_{m+2 r-2}}$. This is because that given a part $\bar{\alpha}_{p}$ with subscript $i$, we always could find a part after $\bar{\alpha}_{p}$ whose subscript is $i+1$.

Let $\gamma$ be a partition having $r$ parts whose parts are $\bar{\alpha}_{t_{0}}, \bar{\alpha}_{t_{2}}, \ldots, \bar{\alpha}_{t_{r-1}}$, respectively. Take $r=2$ in the above example, $\gamma=(3,3)$. We now construct $(\alpha, \beta)$ with $r$ balanced parts and $l(\alpha)-l(\beta)=m$. The partition $\alpha$ consists of all parts of $\bar{\alpha}$ except for the parts in $\gamma$. The partition $\beta$ is composed of all parts of $\bar{\beta}$ and $\gamma$. In the above example, we therefore get $\left(\begin{array}{c}\alpha \\ \beta\end{array}\right)=\left(\begin{array}{ccccccc}6 & 5 & 5 & 5 & 3 & 2 & 1 \\ & 4 & 4 & 3 & \underline{3} & \underline{3} & \end{array}\right)$.

It's obvious to see that $|\bar{\alpha}|+|\bar{\beta}|=|\alpha|+|\beta|, l(\alpha)-l(\beta)=l(\bar{\alpha})-l(\gamma)-[l(\bar{\beta})+l(\gamma)]=$ $m$. We now show that $(\alpha, \beta)$ has exactly $r$ balanced parts. From Lemma 2.5 and the definition of $\gamma$, we know that for each part $\beta_{t}$ from $\gamma$ in $\beta$, the number of parts greater than $\beta_{t}$ in $\alpha$ equals the number of parts from $\bar{\beta}$ greater than or equal to $\beta_{t}$ in $\beta$. Thus we just need to prove that the parts from $\bar{\beta}$ in $\beta$ are unbalanced. We use induction on the parts from $\bar{\beta}$ in $\beta$. We first verify that the largest part $\bar{\beta}_{1}$ of $\bar{\beta}$ is unbalanced in $\beta$. Suppose that there are $t$ parts $\gamma_{1}, \gamma_{2}, \ldots, \gamma_{t}$ from $\gamma$ greater than $\bar{\beta}_{1}$. Then $\gamma_{i}=\bar{\alpha}_{i+1}, i=1,2, \ldots, t$ and $\alpha_{2}=\bar{\alpha}_{t+2}$. We claim that $\alpha_{2}=\bar{\alpha}_{t+2}>\bar{\beta}_{1}$. Recall that the part $\gamma_{i}$ is the smallest part whose subscript is $i-1$. If $\alpha_{2}=\bar{\alpha}_{t+2} \leq \bar{\beta}_{1}$, the subscript of $\bar{\alpha}_{t+2}$ is less than $t$. This contradicts the definition of $\gamma_{i}$. Clearly, these $t$ parts from $\gamma$ are balanced, and $\bar{\beta}_{1}$ is not balanced. We now consider the part $\bar{\beta}_{j}$ from $\bar{\beta}$ in $\beta$. Assume that all parts from $\bar{\beta}$ before $\bar{\beta}_{j}$ in $\beta$ are not balanced and there are $t$ parts from $\gamma$ before $\bar{\beta}_{j}$. We next justify $\alpha_{j+1}>\bar{\beta}_{j}$ and then by the induction hypothesis, we know that there are $j-1$ unbalanced parts before $\bar{\beta}_{j}$ in $\beta$, while there are at least $j$ parts larger than $\bar{\beta}_{j}$ in $\alpha$, so $\bar{\beta}_{j}$ is unbalanced. Since there are $t$ parts from $\gamma$ before $\bar{\beta}_{j}$, then $\alpha_{j+1}=\bar{\alpha}_{t+j+1}$ and if $\bar{\alpha}_{t+j+1}=\alpha_{j+1} \leq \bar{\beta}_{j}$, then the subscript of $\bar{\alpha}_{t+j+1}$ is less than $t$, which contradicts the definition of the parts of $\gamma$, so $\alpha_{j+1}>\bar{\beta}_{j}$. This completes the proof.

The next theorem gives a relation between $k$-marked strict shifted Durfee symbols and $k$-marked Durfee symbols.

Theorem 2.6. Given $2 k$ nonnegative integers $m_{1}, m_{2}, \ldots, m_{k}$, and $t_{1}, t_{2}, \ldots, t_{k}$ where $t_{k}=0$. There is a bijection $\Psi$ between the set of $k$-marked Durfee symbols of $n$ with ith rank equal to $m_{i}$ and ith balanced number equal to $t_{i}$ and the set of $k$-marked strict shifted Durfee symbols of $n$ with ith rank equal to $m_{i}+2 t_{i}$.

Proof. Let $\eta=\left(\begin{array}{cccc}\alpha^{k}, & \alpha^{k-1}, & \ldots, & \alpha^{1} \\ \beta^{k}, & \beta^{k-1}, & \ldots, & \beta^{1}\end{array}\right)_{D}$ be a $k$-marked Durfee symbol with $i$ th rank equal to $m_{i}$ and $i$ th balanced number equal to $t_{i}$. We now apply the bijection $\psi$ in Theorem 2.4 to each vector $\left(\alpha^{i}, \beta^{i}\right)$ of $\eta$ except for the $k$ th vector $\left(\alpha^{k}, \beta^{k}\right)$, and let $\left(\bar{\alpha}^{i}, \bar{\beta}^{i}\right)=\psi\left(\alpha^{i}, \beta^{i}\right)$. From Theorem 2.4, it's known that $\left(\bar{\alpha}^{i}, \bar{\beta}^{i}\right)$ is strict shifted and $l\left(\bar{\alpha}^{i}\right)-l\left(\bar{\beta}^{i}\right)=l\left(\alpha^{i}\right)-l\left(\beta^{i}\right)+2 t_{i}$. Define $\bar{\eta}=\left(\begin{array}{cccc}\alpha^{k}, & \bar{\alpha}^{k-1}, & \ldots, & \bar{\alpha}^{1} \\ \beta^{k}, & \bar{\beta}^{k-1}, & \ldots, & \bar{\beta}^{1}\end{array}\right)_{D}$, which has the same subscript and the same $k$ th vector with $\eta$. It's obvious to see that $\bar{\eta}$ is a $k$-marked strict shifted Durfee symbol with $i$ th rank equal to $m_{i}+2 t_{i}$.

By Theorem 2.6, one can derive the following identity readily. 
Corollary 2.7. For $m_{i} \geq 0$ and $k \geq 2$, we have

$$
\mathcal{D}_{k}\left(m_{1}, m_{2}, \ldots, m_{k} ; n\right)=\sum_{t_{1}, \ldots, t_{k-1}=0}^{+\infty} \mathcal{D}_{k}^{s s}\left(m_{1}+2 t_{1}, \ldots, m_{k-1}+2 t_{k-1}, m_{k} ; n\right) .
$$

Combine Corollaries 2.2 and 2.7 to get:

Theorem 2.8. For $m_{i} \geq 0$ and $k \geq 2$, we have

$$
\mathcal{D}_{k}\left(m_{1}, m_{2}, \ldots, m_{k} ; n\right)=\sum_{t_{1}, \ldots, t_{k-1}=0}^{+\infty} N\left(\sum_{i=1}^{k} m_{i}+2 \sum_{i=1}^{k-1} t_{i}+k-1 ; n\right) .
$$

The following compact form of Theorem 2.8 can be easily obtained upon utilizing the fact that the number of solutions to $t_{1}+t_{2}+\cdots+t_{k-1}=j$ in nonnegative integers is $\left(\begin{array}{c}j+k-2 \\ k-2\end{array}\right)$.

Theorem 2.9. For $m_{i} \geq 0$ and $k \geq 2$, we have

$$
\mathcal{D}_{k}\left(m_{1}, m_{2}, \ldots, m_{k} ; n\right)=\sum_{j=0}^{+\infty}\left(\begin{array}{c}
j+k-2 \\
k-2
\end{array}\right) N\left(\sum_{i=1}^{k} m_{i}+2 j+k-1 ; n\right) .
$$

We next generalize Theorem 2.9 to give Theorem 1.5, which holds for any integer $m_{i}$. To do this, we prove the following conclusion by constructing a simple bijection $\Theta$.

Theorem 2.10. For $k \geq 1$ and $1 \leq p \leq k$, we have

$$
\mathcal{D}_{k}\left(m_{1}, \ldots, m_{p}, \ldots, m_{k} ; n\right)=\mathcal{D}_{k}\left(m_{1}, \ldots,-m_{p}, \ldots, m_{k} ; n\right) .
$$

Proof. Let $\eta=\left(\begin{array}{cccc}\alpha^{k}, & \alpha^{k-1}, & \ldots, & \alpha^{1} \\ \beta^{k}, & \beta^{k-1}, & \ldots, & \beta^{1}\end{array}\right)$ be a $k$-marked Durfee symbol with $i$ th rank equal to $m_{i}$. We will construct another $k$-marked Durfee symbol $\bar{\eta}$ with $i$ th rank equal to $\bar{m}_{i}$ such that $\bar{m}_{p}=-m_{p}$ and $\bar{m}_{i}=m_{i}$ for $i \neq p$.

Define

$$
\bar{\eta}=\left(\begin{array}{ccccccc}
\alpha^{k}, & \ldots, & \alpha^{p+1} & \bar{\alpha}^{p}, & \alpha^{p-1}, & \ldots, & \alpha^{1} \\
\beta^{k}, & \ldots, & \beta^{p+1}, & \bar{\beta}^{p}, & \beta^{p-1}, & \ldots, & \beta^{1}
\end{array}\right)_{D},
$$

where $\bar{\alpha}^{k}=\beta^{k}$ and $\bar{\beta}^{k}=\alpha^{k}$ for $p=k$. When $p \neq k, \bar{\alpha}^{p}$ consists of all parts of $\beta^{p}$ and the largest part $\alpha_{1}^{p}$ of $\alpha^{p}$. $\bar{\beta}^{p}$ consists of all parts of $\alpha^{p}$ except for the largest part $\alpha_{1}^{p}$. It's clear to see that $\bar{m}_{p}=l\left(\bar{\alpha}^{p}\right)-l\left(\bar{\beta}^{p}\right)-1=l\left(\beta^{p}\right)+1-\left[l\left(\alpha^{p}\right)-1\right]-1=$ $\left.-\left[l\left(\alpha^{p}\right)-l\left(\beta^{p}\right)-1\right)\right]=-m_{p}$ for $p \neq k$ and $\bar{m}_{k}=l\left(\bar{\alpha}^{k}\right)-l\left(\bar{\beta}^{k}\right)=-\left[l\left(\alpha^{k}\right)-l\left(\beta^{k}\right)\right]=$ $-m_{k}$, so $\bar{\eta}$ is as desired.

By Theorem 2.10, we could generalize Theorem 2.8 to give the following theorem which is useful to prove a relationship between $k$-marked Durfee symbols and the symmetrized rank moment given by Andrews (see Theorem 3.3).

Theorem 2.11. For $k \geq 2$, we have

$$
\mathcal{D}_{k}\left(m_{1}, m_{2}, \ldots, m_{k} ; n\right)=\sum_{t_{1}, \ldots, t_{k-1}=0}^{+\infty} N\left(\sum_{i=1}^{k}\left|m_{i}\right|+2 \sum_{i=1}^{k-1} t_{i}+k-1 ; n\right) .
$$

Theorem 1.5 is a compact form of Theorem 2.11, which immediately follows from Theorems 2.9 and 2.10 . 


\section{Andrews' identities on $k$-MARKed Durfee Symbols}

In this section, we aim to show the identities on $k$-marked Durfee symbols given by Andrews with the help of Theorem 1.5. Recall that $\mathcal{D}_{k}\left(m_{1}, m_{2}, \ldots, m_{k} ; n\right)$ denotes the number of $k$-marked Durfee symbols of $n$ with $i$ th rank equal to $m_{i}$. Andrews considered the following generating function for $\mathcal{D}_{k}\left(m_{1}, m_{2}, \ldots, m_{k} ; n\right)$ :

$$
R_{k}\left(x_{1}, \ldots, x_{k} ; q\right)=\sum_{m_{1}, \ldots, m_{k}=-\infty}^{+\infty} \sum_{n=0}^{+\infty} \mathcal{D}_{k}\left(m_{1}, \ldots, m_{k} ; n\right) x_{1}^{m_{1}} \cdots x_{k}^{m_{k}} q^{n},|q|<1 .
$$

By applying the $k$-fold generalization of Watson's transformation between a verywell-poised ${ }_{8} \phi_{7}$-series and a balanced ${ }_{4} \phi_{3}$-series [1, p.199, Theorem 4], Andrews gave the explicit form of the generating function $R_{k}\left(x_{1}, x_{2}, \ldots, x_{k} ; q\right)$ in the following theorem.

Theorem 3.1 (Corollary 11, Andrews [4]).

$$
R_{k}\left(x_{1}, x_{2}, \ldots, x_{k} ; q\right)=\frac{1}{(q ; q)_{\infty}} \sum_{n=1}^{+\infty}(-1)^{n-1} q^{3 n(n-1) / 2+k n} \frac{\left(1+q^{n}\right)\left(1-q^{n}\right)^{2}}{\prod_{j=1}^{k}\left(1-x_{j} q^{n}\right)\left(1-\frac{q^{n}}{x_{j}}\right)} .
$$

Proof. We will reformulate this identity as the partition identity (1.7) in Theorem 1.5. The key step is to give a partition interpretation of the right hand side of (3.2). We will show that it is the generating function for the summation on the right hand side of (1.7).

First, the right hand side of (3.2) can be written as the difference between the following two terms:

$$
\begin{aligned}
& \frac{1}{(q ; q)_{\infty}} \sum_{n=1}^{+\infty}(-1)^{n} q^{n(3 n-1) / 2+k n} \frac{\left(1-x_{1}\right)\left(1-x_{1}^{-1}\right)\left(1+q^{n}\right)}{\left(1-x_{1} q^{n}\right)\left(1-x_{1}^{-1} q^{n}\right)} \\
& \times \frac{1}{\prod_{j=2}^{k}\left(1-x_{j} q^{n}\right)\left(1-\frac{q^{n}}{x_{j}}\right)} \\
&-\frac{1}{(q ; q)_{\infty}} \sum_{n=1}^{+\infty}(-1)^{n} q^{n(3 n-1) / 2+(k-1) n} \frac{1+q^{n}}{\prod_{j=2}^{k}\left(1-x_{j} q^{n}\right)\left(1-\frac{q^{n}}{x_{j}}\right)} .
\end{aligned}
$$

We next expand each term of the above two terms, noting that

$$
\begin{aligned}
\frac{\left(1-x_{1}\right)\left(1-x_{1}^{-1}\right)\left(1+q^{n}\right)}{\left(1-x_{1} q^{n}\right)\left(1-x_{1}^{-1} q^{n}\right)} & =\frac{\left(1-x_{1}\right)}{\left(1-x_{1} q^{n}\right)}+\frac{\left(1-x_{1}^{-1}\right)}{\left(1-x_{1}^{-1} q^{n}\right)} \\
& =2+\sum_{\substack{m_{1}=-\infty \\
m_{1} \neq 0}}^{+\infty} x_{1}^{m_{1}}\left[q^{n\left|m_{1}\right|}-q^{n\left(\left|m_{1}\right|-1\right)}\right], \\
\frac{1}{\left(1-x_{j} q^{n}\right)\left(1-x_{j}^{-1} q^{n}\right)} & =\sum_{a=0}^{+\infty} x_{j}^{a} q^{n a} \sum_{b=0}^{+\infty} x_{j}^{-b} q^{n b}=\sum_{m_{j}=-\infty}^{+\infty} x_{j}^{m_{j}} \sum_{t_{j}=0}^{+\infty} q^{n\left(\left|m_{j}\right|+2 t_{j}\right)} .
\end{aligned}
$$


Given $k$ integers $m_{1} \ldots, m_{k}$, it's clear to see that the coefficients of $x_{1}^{m_{1}} x_{2}^{m_{2}} \cdots x_{k}^{m_{k}}$ on the series expansion of the right hand side of (3.2) are

$$
\begin{aligned}
& \frac{1}{(q ; q)_{\infty}} \sum_{n=1}^{+\infty}(-1)^{n} q^{n(3 n-1) / 2+k n}\left[q^{n\left|m_{1}\right|}-q^{n\left(\left|m_{1}\right|-1\right)}\right] \sum_{t_{2}, \ldots, t_{k}=0}^{+\infty} q^{n\left(\sum_{i=2}^{k}\left|m_{i}\right|+2 \sum_{i=2}^{k} t_{i}\right)} \\
= & \frac{1}{(q ; q)_{\infty}} \sum_{n=1}^{+\infty}(-1)^{n-1} q^{n(3 n-1) / 2+k n}\left(1-q^{n}\right) q^{n\left(\left|m_{1}\right|-1\right)} \sum_{j=0}^{+\infty}\left(\begin{array}{c}
j+k-2 \\
k-2
\end{array}\right) q^{n\left(\sum_{i=2}^{k}\left|m_{i}\right|+2 j\right)} \\
= & \sum_{j=0}^{+\infty}\left(\begin{array}{c}
j+k-2 \\
k-2
\end{array}\right) \frac{1}{(q ; q)_{\infty}} \sum_{n=1}^{+\infty}(-1)^{n-1} q^{n(3 n-1) / 2}\left(1-q^{n}\right) q^{n\left(\sum_{i=1}^{k}\left|m_{i}\right|+2 j+k-1\right)} \\
= & \sum_{j=0}^{+\infty}\left(\begin{array}{c}
j+k-2 \\
k-2
\end{array}\right) \sum_{n=0}^{+\infty} N\left(\sum_{i=1}^{k}\left|m_{i}\right|+2 j+k-1 ; n\right) q^{n} \\
= & \sum_{n=0}^{+\infty} q^{n}\left[\begin{array}{c}
+\infty \\
j=0
\end{array}\left(\begin{array}{c}
j+k-2 \\
k-2
\end{array}\right) N\left(\sum_{i=1}^{k}\left|m_{i}\right|+2 j+k-1 ; n\right)\right]
\end{aligned}
$$

where the penultimate identity follows from Theorem [1.1, and we then obtain the following combinatorial interpretation:

$$
\frac{1}{(q ; q)_{\infty}} \sum_{n=1}^{+\infty}(-1)^{n-1} q^{3 n(n-1) / 2+k n} \frac{\left(1+q^{n}\right)\left(1-q^{n}\right)^{2}}{\prod_{j=1}^{k}\left(1-x_{j} q^{n}\right)\left(1-\frac{q^{n}}{x_{j}}\right)}
$$

$=\sum_{m_{1}, \ldots, m_{k}=-\infty}^{+\infty} \sum_{n=0}^{+\infty}\left[\sum_{j=0}^{+\infty}\left(\begin{array}{c}j+k-2 \\ k-2\end{array}\right) N\left(\sum_{i=1}^{k}\left|m_{i}\right|+2 j+k-1 ; n\right)\right] x_{1}^{m_{1}} \cdots x_{k}^{m_{k}} q^{n}$.

Combining (3.1) and (3.3), we reach our conclusion that the identity (3.2) can be restated as the partition identity (1.7). Thus, we have obtained a combinatorial proof of (3.2) based on Theorem 1.5.

Recently, Bringmann, Lovejoy, and Osburn defined a two-parameter generalization of $k$-marked Durfee symbols in 11. They deduced the generating function 11. Theorem 2.2] for the two-parameter generalization of $k$-marked Durfee symbols using a similar argument of Andrews, which reduces to the identity (3.2) when $d=e=0$.

From the generating function $R_{k}\left(x_{1}, x_{2}, \ldots, x_{k} ; q\right)$ in Theorem 3.1. Andrews immediately found the following symmetry of $k$-marked Durfee symbols.

Theorem 3.2 (Corollary 12, Andrews [4]). $\mathcal{D}_{k}\left(m_{1}, m_{2}, \ldots, m_{k} ; n\right)$ is symmetric in $m_{1}, m_{2}, \ldots, m_{k}$.

Proof. This symmetry can also immediately follow from Theorem 1.5 .

In fact, the composite of the bijections in Section 2 provides a bijection for this symmetry. We give an example to explain this process. Letting

$$
\eta=\left(\begin{array}{llllllll}
6_{3} & 3_{2} & 3_{2} & 2_{2} & 2_{2} & 1_{2} & 1_{1} & \\
5_{3} & 3_{2} & 3_{2} & 1_{2} & & & 1_{1} & 1_{1}
\end{array}\right)_{6} \text { be counted by } \mathcal{D}_{3}(-2,1,0 ; 68)
$$


we aim to construct a 3 -marked Durfee symbol $\bar{\eta}$ counted by $\mathcal{D}_{3}(1,-2,0 ; 68)$. We will first combine all subscripts of the 3-marked Durfee symbol $\eta$ to get a Durfee symbol, and then split this Durfee symbol over again to get our desired 3-marked Durfee symbol $\bar{\eta}$.

First, applying the bijection $\Theta$ in Theorem 2.10 to $\eta$, we get a 3-marked Durfee symbol $\eta^{\prime}$ which is enumerated by $\mathcal{D}_{3}(2,1,0 ; 68)$,

$$
\eta^{\prime}=\left(\begin{array}{lllllllll}
6_{3} & 3_{2} & 3_{2} & 2_{2} & 2_{2} & 1_{2} & 1_{1} & 1_{1} & 1_{1} \\
5_{3} & & \underline{3_{2}} & \underline{3_{2}} & 1_{2} & & & &
\end{array}\right)_{6} .
$$

Next, utilize the bijection $\Psi$ in Theorem 2.6 to get a 3-marked strict shifted Durfee symbol $\eta^{\prime \prime}$. Observe that there are two balanced parts in the second vector of $\eta^{\prime}$, and there is no balanced part in other vectors of $\eta^{\prime}$. So we will get a 3-marked strict shifted Durfee symbol $\eta^{\prime \prime}$ which is counted by $\mathcal{D}_{3}^{s s}(2,1+2 \times 2,0 ; 68)$,

$$
\eta^{\prime \prime}=\left(\begin{array}{lllllllllll}
6_{3} & 3_{2} & 3_{2} & 3_{2} & 3_{2} & 2_{2} & 2_{2} & 1_{2} & 1_{1} & 1_{1} & 1_{1} \\
5_{3} & & 1_{2} & & & & & & & &
\end{array}\right)_{6} .
$$

We then apply the bijection $\Phi$ in Theorem 2.1 to get a Durfee symbol $\eta^{\prime \prime \prime}$ which is counted by $\mathcal{D}_{1}(9 ; 68)$,

$$
\eta^{\prime \prime \prime}=\left(\begin{array}{rrrrrrrrrrr}
6 & 3 & 3 & 3 & 3 & 2 & 2 & 1 & 1 & 1 & 1 \\
5 & 1 & & & & & & & & &
\end{array}\right)_{6} .
$$

Thus we complete the first step. We next split the Durfee symbol $\eta^{\prime \prime \prime}$ over again.

First, apply the inverse map $\Phi^{-1}$ in Theorem 2.1 to get a 3-marked strict shifted Durfee symbol $\bar{\eta}^{\prime \prime}$, which is counted by $\mathcal{D}_{3}^{s s}(1,2+2 \times 2,0 ; 68)$,

$$
\bar{\eta}^{\prime \prime}=\left(\begin{array}{lllllllllll}
6_{3} & 3_{2} & \underline{3_{2}} & \underline{3_{2}} & 3_{2} & 2_{2} & 2_{2} & 1_{2} & 1_{2} & 1_{1} & 1_{1} \\
5_{3} & 1_{2} & & & & & & & & &
\end{array}\right)_{6} .
$$

Using the inverse map $\Psi^{-1}$ in Theorem 2.6, we get a 3-marked Durfee symbol $\bar{\eta}^{\prime}$ counted by $\mathcal{D}_{3}(1,2,0 ; 68)$,

$$
\bar{\eta}^{\prime}=\left(\begin{array}{lllllllll}
6_{3} & 3_{2} & 3_{2} & 2_{2} & 2_{2} & 1_{2} & 1_{2} & 1_{1} & 1_{1} \\
5_{3} & & 3_{2} & 3_{2} & 1_{2} & & & &
\end{array}\right)_{6} .
$$

Finally, the desired 3 -marked Durfee symbol $\bar{\eta}$ counted by $\mathcal{D}_{3}(1,-2,0 ; 68)$ is obtained when we apply the bijection $\Theta$ in Theorem 2.10 to $\bar{\eta}^{\prime}$,

$$
\bar{\eta}=\left(\begin{array}{llllllll}
6_{3} & 3_{2} & 3_{2} & 3_{2} & 1_{2} & & 1_{1} & 1_{1} \\
5_{3} & 3_{2} & 2_{2} & 2_{2} & 1_{2} & 1_{2} & &
\end{array}\right)_{6} .
$$

By the generating function $R_{k}\left(x_{1}, x_{2}, \ldots, x_{k} ; q\right)$ and the generating function for $\eta_{2 k}(n)$, Andrews showed that the number of $(k+1)$-marked Durfee symbols of $n$ equals the symmetrized $(2 k)$-th moment function at $n$ in [4], that is:

Theorem 3.3 (Corollary 13, Andrews [4]). For $k \geq 1$,

$$
\mathcal{D}_{k+1}(n)=\eta_{2 k}(n) \text {. }
$$

Proof. Recall that

$$
\eta_{2 k}(n)=\sum_{m=-\infty}^{+\infty}\left(\begin{array}{c}
m+k-1 \\
2 k
\end{array}\right) N(m ; n)=\sum_{m=1}^{+\infty}\left[\left(\begin{array}{c}
m+k-1 \\
2 k
\end{array}\right)+\left(\begin{array}{c}
m+k \\
2 k
\end{array}\right)\right] N(m ; n),
$$


where the second equality follows from the rank symmetry $N(-m ; n)=N(m ; n)$ and the fact that $\left(\begin{array}{c}-m+k-1 \\ 2 k\end{array}\right)=\left(\begin{array}{c}m+k \\ 2 k\end{array}\right)$, while

$$
\begin{aligned}
\mathcal{D}_{k+1}(n) & =\sum_{m_{1}, \ldots, m_{k+1}=-\infty}^{+\infty} \mathcal{D}_{k+1}\left(m_{1}, m_{2}, \ldots, m_{k+1} ; n\right) \\
& =\sum_{m_{1}, \ldots, m_{k+1}=-\infty}^{+\infty} \sum_{t_{1}, \ldots, t_{k}=0}^{+\infty} N\left(\sum_{i=1}^{k+1}\left|m_{i}\right|+2 \sum_{i=1}^{k} t_{i}+k ; n\right),
\end{aligned}
$$

where the second equality follows from Theorem 2.11 So it suffices to show that the number of solutions to $\left|m_{1}\right|+\cdots+\left|m_{k+1}\right|+2 t_{1}++\cdots+2 t_{k}=m-k$, where $m_{i}$ is an integer, and $t_{i}$ is a nonnegative integer is equal to $\left(\begin{array}{c}m+k-1 \\ 2 k\end{array}\right)+\left(\begin{array}{c}m+k \\ 2 k\end{array}\right)$.

Let $c(n)$ denote the number of solutions to $\left|m_{1}\right|+\left|m_{2}\right|+\cdots+\left|m_{k+1}\right|+2 t_{1}+$ $2 t_{2}+\cdots+2 t_{k}=n$, where $m_{i}$ is an integer and $t_{i}$ is a nonnegative integer. It's easy to know that the generating function for $c(n)$ is

$$
\begin{aligned}
\sum_{n=0}^{+\infty} c(n) q^{n} & =\left(1+2 q+2 q^{2}+2 q^{3}+\cdots\right)^{k+1}\left(1+q^{2}+q^{4}+q^{6}+\cdots\right)^{k} \\
& =\frac{(1+q)^{k+1}}{(1-q)^{k+1}} \times \frac{1}{\left(1-q^{2}\right)^{k}} \\
& =(1+q) \times \frac{1}{(1-q)^{2 k+1}} \\
& =(1+q) \sum_{n=0}^{+\infty}\left(\begin{array}{c}
2 k+n \\
n
\end{array}\right) q^{n} \\
& =\sum_{n=0}^{+\infty}\left(\begin{array}{c}
2 k+n \\
2 k
\end{array}\right) q^{n}+\sum_{n=1}^{+\infty}\left(\begin{array}{c}
2 k+n-1 \\
2 k
\end{array}\right) q^{n} .
\end{aligned}
$$

Comparing the coefficients of $q^{n}$ in the above expression, we obtain

$$
c(n)=\left(\begin{array}{c}
2 k+n \\
2 k
\end{array}\right)+\left(\begin{array}{c}
2 k+n-1 \\
2 k
\end{array}\right) .
$$

Thus we reach our conclusion.

By partial fraction expansion, Andrews [4] also gave the following relationship between the generating function for $k$-marked Durfee symbols and the generating function for Durfee symbols, which plays an important role in the study of Ramanujan-type congruences for $k$-marked Durfee symbols.

Theorem 3.4 (Theorem 7, Andrews [4).

$$
R_{k}\left(x_{1}, x_{2}, \ldots, x_{k} ; q\right)=\sum_{i=1}^{k} \frac{R_{1}\left(x_{i} ; q\right)}{\prod_{\substack{j=1 \\ j \neq i}}^{k}\left(x_{i}-x_{j}\right)\left(1-\frac{1}{x_{i} x_{j}}\right)} .
$$

Proof. Similarly, we will restate this identity as the partition identity (1.7) in Theorem 1.5. We first consider the series expansion of the right hand side of (3.5). To do this, we need to work in a larger ring: the field of iterated Laurent series $K\left\langle\left\langle x_{k}, x_{k-1}, \ldots, x_{1}\right\rangle\right\rangle=K\left(\left(x_{k}\right)\right)\left(\left(x_{k-1}\right)\right) \cdots\left(\left(x_{1}\right)\right)$, where $K=\mathbb{C}(q)$, in which all series are regarded first as Laurent series in $x_{1}$, then as Laurent series in $x_{2}$, 
and so on. For a more detailed account of the properties of this field, with other applications, see [18] and [19].

Every element of $K\left\langle\left\langle x_{k}, x_{k-1}, \ldots, x_{1}\right\rangle\right\rangle$ has a unique iterated Laurent series expansion. In particular, the series expansion of $1 /\left(1-\frac{1}{x_{i} x_{j}}\right)$ is:

$$
\frac{1}{1-\frac{1}{x_{i} x_{j}}}=-\frac{x_{i} x_{j}}{1-x_{i} x_{j}}=-\sum_{l=1}^{+\infty} x_{i}^{l} x_{j}^{l}
$$

The series expansion of $1 /\left(x_{i}-x_{j}\right)$ will be especially important. If $j<i$, then

$$
\frac{1}{x_{i}-x_{j}}=\frac{x_{i}^{-1}}{1-\frac{x_{j}}{x_{i}}}=\sum_{l=0}^{+\infty} x_{i}^{-l-1} x_{j}^{l}
$$

However, if $j>i$, then this expansion is not valid and instead we have the expansion:

$$
\frac{1}{x_{i}-x_{j}}=\frac{-x_{j}^{-1}}{1-\frac{x_{i}}{x_{j}}}=-\sum_{l=0}^{+\infty} x_{i}^{l} x_{j}^{-l-1} .
$$

Thus for $j<i$, the series expansion of $\frac{1}{\left(x_{i}-x_{j}\right)\left(1-x_{i}^{-1} x_{j}^{-1}\right)}$ is

$$
\frac{1}{\left(x_{i}-x_{j}\right)\left(1-x_{i}^{-1} x_{j}^{-1}\right)}=-\sum_{l=1}^{+\infty} x_{i}^{l} x_{j}^{l} \sum_{m=0}^{+\infty} x_{i}^{-m-1} x_{j}^{m}=-\sum_{m_{j}=1}^{+\infty} x_{j}^{m_{j}} \sum_{t_{j}=0}^{m_{j}-1} x_{i}^{2 t_{j}-m_{j}+1} \text {, }
$$

and for $j>i$, we have the following expansion:

$$
\frac{1}{\left(x_{i}-x_{j}\right)\left(1-x_{i}^{-1} x_{j}^{-1}\right)}=\sum_{l=1}^{+\infty} x_{i}^{l} x_{j}^{l} \sum_{m=0}^{+\infty} x_{i}^{m} x_{j}^{-m-1}=\sum_{m_{j}=-\infty}^{+\infty} x_{j}^{m_{j}} \sum_{t_{j}=0}^{+\infty} x_{i}^{\left|m_{j}\right|+2 t_{j}+1} .
$$

We now consider the series expansion of the $i$ th term of the right hand side of (3.5):

$$
\frac{R_{1}\left(x_{i} ; q\right)}{\prod_{\substack{j=1 \\ j \neq i}}^{k}\left(x_{i}-x_{j}\right)\left(1-\frac{1}{x_{i} x_{j}}\right)}=\frac{\sum_{n=0}^{+\infty} \sum_{m_{i}=-\infty}^{+\infty} \mathcal{D}_{1}\left(m_{i} ; n\right) x_{i}^{m_{i}} q^{n}}{\prod_{\substack{j=1 \\ j \neq i}}^{k}\left(x_{i}-x_{j}\right)\left(1-\frac{1}{x_{i} x_{j}}\right)}
$$

By (3.7) and (3.8), we obtain a series expansion of (3.9), in which the exponents of $x_{1}, x_{2}, \ldots, x_{i-1}$ must be positive and the coefficients of $x_{1}^{m_{1}} \cdots x_{k}^{m_{k}} q^{n}$ for $m_{1}, \ldots, m_{i-1} \geq 1$ are

$$
(-1)^{i-1} \sum_{\mathbf{t}_{i}} \mathcal{D}_{1}\left(\sum_{j=1}^{i-1} m_{j}+m_{i}-\sum_{j=i+1}^{k}\left|m_{j}\right|-2\left|\mathbf{t}_{i}\right|-(k-1) ; n\right),
$$

where the sum ranges over all sequences $\mathbf{t}_{i}=\left(t_{1}, \ldots, \hat{t}_{i}, \ldots, t_{k}\right.$ ) (omitting $t_{i}$ ) where $0 \leq t_{j}<\left|m_{j}\right|$ for $j<i$ and $t_{j}$ could be an arbitrary nonnegative integer for $j>i$. Define $\left|\mathbf{t}_{i}\right|=\sum_{\substack{j=1 \\ j \neq i}}^{k} t_{j}$. 
Thus, the right hand side of (3.5) has the following series expansion:

$$
\begin{gathered}
\sum_{i=1}^{k} \frac{R_{1}\left(x_{i} ; q\right)}{\prod_{\substack{j=1 \\
j \neq i}}^{k}\left(x_{i}-x_{j}\right)\left(1-\frac{1}{x_{i} x_{j}}\right)}=\sum_{i=1}^{k} \sum_{m_{1}, \ldots, m_{i-1}=1}^{+\infty} \sum_{m_{i}, \ldots, m_{k}=-\infty}^{+\infty} \sum_{n=0}^{+\infty} x_{1}^{m_{1}} \cdots x_{k}^{m_{k}} q^{n} \\
\times\left[(-1)^{i-1} \sum_{\mathbf{t}_{i}} \mathcal{D}_{1}\left(\sum_{j=1}^{i-1} m_{j}+m_{i}-\sum_{j=i+1}^{k}\left|m_{j}\right|-2\left|\mathbf{t}_{i}\right|-(k-1) ; n\right)\right] .
\end{gathered}
$$

Let $\mathbf{m}_{i}=\left(m_{1}, \ldots, m_{k}\right)$, where $m_{j} \geq 1$ for $j<i, m_{i} \leq 0$, and others could be arbitrary integers. Define $\mathbf{x}^{\mathbf{m}_{i}}=x_{1}^{m_{1}} \cdots x_{k}^{m_{k}}$. Obviously, the term $\mathbf{x}^{\mathbf{m}_{i}} q^{n}$ would have appeared in the first $i$ terms of (3.10). We next use induction to prove that the sum of coefficients of $\mathbf{x}^{\mathbf{m}_{i}} q^{n}$ in (3.10) equals

$$
\sum_{j=0}^{+\infty}\left(\begin{array}{c}
j+k-2 \\
k-2
\end{array}\right) N\left(\sum_{i=1}^{k}\left|m_{i}\right|+2 j+k-1 ; n\right) .
$$

We first consider the coefficients of $\mathbf{x}^{\mathbf{m}_{1}} q^{n}$ in (3.10). It's known that only the first term of (3.10) contains the term $\mathbf{x}^{\mathbf{m}_{1}} q^{n}$, and the coefficients of $\mathbf{x}^{\mathbf{m}_{1}} q^{n}$ are

$$
\begin{aligned}
& \sum_{\mathbf{t}_{1}} \mathcal{D}_{1}\left(m_{1}-\sum_{i=2}^{k}\left|m_{i}\right|-2\left|\mathbf{t}_{1}\right|-(k-1) ; n\right) \\
& =\sum_{j=0}^{+\infty}\left(\begin{array}{c}
j+k-2 \\
k-2
\end{array}\right) N\left(\sum_{i=1}^{k}\left|m_{i}\right|+2 j+k-1 ; n\right),
\end{aligned}
$$

where the equality follows from the fact that $m_{1} \leq 0, \mathcal{D}_{1}(-m ; n)=N(m ; n)$ and the number of solutions to $t_{2}+t_{3}+\cdots+t_{k}=j$ in nonnegative integers is $\left(\begin{array}{c}j+k-2 \\ k-2\end{array}\right)$.

Assuming that the sum of coefficients of $\mathbf{x}^{\mathbf{m}_{p}} q^{n}$ in (3.10) equals (3.11), we now show that the sum of coefficients of $\mathbf{x}^{\mathbf{m}_{p+1}} q^{n}$ is also equal to (3.11). Observe that $\mathbf{x}^{\mathbf{m}_{p}} q^{n}$ appears in the first $p$ terms of (3.10) and the term $\mathbf{x}^{\mathbf{m}_{p+1}} q^{n}$ appears in the first $(p+1)$ terms. Furthermore, the coefficients of $\mathbf{x}^{\mathbf{m}_{p}} q^{n}$ and $\mathbf{x}^{\mathbf{m}_{p+1}} q^{n}$ are the same in the first $(p-1)$ terms. Therefore, if we verify the sum of the coefficients of $\mathbf{x}^{\mathbf{m}_{p+1}} q^{n}$ in the $p$ th term and the $(p+1)$ th term of (3.10) is equal to the coefficients of $\mathbf{x}^{\mathbf{m}_{p}} q^{n}$ in the $p$ th term, we could reach our conclusion by the induction hypothesis.

The coefficients of $\mathbf{x}^{\mathbf{m}_{p}} q^{n}$ (where $m_{p} \leq 0$ ) in the $p$ th term of (3.10) are

$$
(-1)^{p-1} \sum_{\mathbf{t}_{p}} \mathcal{D}_{1}\left(\sum_{j=1}^{p-1} m_{j}-\sum_{j=p}^{k}\left|m_{j}\right|-2\left|\mathbf{t}_{p}\right|-(k-1) ; n\right),
$$

and the sum of the coefficients of $\mathbf{x}^{\mathbf{m}_{p+1}} q^{n}$ (where $m_{p} \geq 1$ ) in the $p$ th term and the $(p+1)$ th term of (3.10) is

$$
\begin{aligned}
& (-1)^{p-1} \sum_{\mathbf{t}_{p}} \mathcal{D}_{1}\left(\sum_{j=1}^{p} m_{j}-\sum_{j=p+1}^{k}\left|m_{j}\right|-2\left|\mathbf{t}_{p}\right|-(k-1) ; n\right) \\
& +(-1)^{p} \sum_{\mathbf{t}_{p+1}} \mathcal{D}_{1}\left(\sum_{j=1}^{p} m_{j}-\sum_{j=p+1}^{k}\left|m_{j}\right|-2\left|\mathbf{t}_{p+1}\right|-(k-1) ; n\right) .
\end{aligned}
$$


In order to prove that the sum (3.13) is equal to (3.12), we need the following lemma.

Lemma 3.5. Given a positive integer $k$ and $p(<k)$ positive integers $m_{1}, m_{2}, \ldots$, $m_{p}$, define $7_{k}^{p}\left(m_{1}, \ldots, m_{p-1}\right)$ to be the set of all sequences $\mathbf{t}_{p}=\left(t_{1}, \ldots, \hat{t}_{p}, \ldots, t_{k}\right)$ of length $k-1$ in nonnegative integers (omitting the pth vector $t_{p}$ ) such that $t_{j}$ is less than $\left|m_{j}\right|$ for $j<p$. Let $\left|\mathbf{t}_{p}\right|=\sum_{\substack{j=1 \\ j \neq p}}^{k} t_{j}$. We have the following two results:

(1) The number of sequences $\left.\mathbf{t}_{p} \in\right\rceil_{k}^{p}\left(m_{1}, \ldots, m_{p-1}\right)$, where the $(p+1)$ th vector $t_{p+1}<\left|m_{p}\right|$, is equal to the number of sequences $\mathbf{t}_{p+1}^{\prime} \in 7_{k}^{p+1}\left(m_{1}, \ldots, m_{p}\right)$ such that $\left|\mathbf{t}_{p}\right|=\left|\mathbf{t}_{p+1}^{\prime}\right|$.

(2) The number of sequences $\left.\mathbf{t}_{p} \in\right\rceil_{k}^{p}\left(m_{1}, \ldots, m_{p-1}\right)$, where the $(p+1)$ th vector $t_{p+1} \geq\left|m_{p}\right|$, is equal to the number of sequences $\mathbf{t}_{p}^{\prime} \in \tau_{k}^{p}\left(m_{1}, \ldots, m_{p-1}\right)$ such that $\left|\mathbf{t}_{p}\right|=\left|\mathbf{t}_{p}^{\prime}\right|+\left|m_{p}\right|$.

Proof. Given a sequence $\mathbf{t}_{p}=\left(t_{1}, \ldots, \hat{t}_{p}, \ldots, t_{k}\right)$ (omitting $t_{p}$ ) where $0 \leq t_{j}<\left|m_{j}\right|$ for $j<p$ and others could be arbitrary nonnegative integers.

(1) If $t_{p+1}<\left|m_{p}\right|$, we define $\mathbf{t}_{p+1}^{\prime}=\left(t_{1}^{\prime}, \ldots, \hat{t}_{p+1}^{\prime}, \ldots, t_{k}^{\prime}\right)$, where $t_{j}^{\prime}=t_{j}$ for $j \neq p, p+1$ and $t_{p}^{\prime}=t_{p+1}$. Obviously, $\mathbf{t}_{p+1}^{\prime} \in \mathcal{T}_{k}^{p+1}\left(m_{1}, \ldots, m_{p}\right)$ and $\left|\mathbf{t}_{p}^{\prime}\right|=\left|\mathbf{t}_{p+1}^{\prime}\right|$.

(2) If $t_{p+1} \geq\left|m_{p}\right|$, we define $\mathbf{t}_{p}^{\prime}=\left(t_{1}^{\prime}, \ldots, \hat{t}_{p}^{\prime}, \ldots, t_{k}^{\prime}\right)$ where $t_{j}^{\prime}=t_{j}$ for $j \neq p+1$ and $t_{p+1}^{\prime}=t_{p+1}-\left|m_{p}\right|$. It's clear to see that $\mathbf{t}_{p}^{\prime} \in \tau_{k}^{p}\left(m_{1}, \ldots, m_{p-1}\right)$ and $\left|\mathbf{t}_{p}\right|=\left|\mathbf{t}_{p}^{\prime}\right|+\left|m_{p}\right|$.

Furthermore, one can easily see that the above two processes are reversible.

By Lemma 3.5 the sum (3.13) is clearly equal to (3.12). Thus we get our conclusion, and by the definition of $R_{k}\left(x_{1}, x_{2}, \ldots, x_{k} ; q\right)$, we could recast (3.5) as the partition identity (1.7).

\section{4. $k$-MARKED ODD DURFEE SYMBOLS}

This section is devoted to solving the problems raised by Andrews (4, Problems $6-9]$ ) on $k$-marked odd Durfee symbols. We begin this section by defining odd Durfee symbols which resemble ordinary Durfee symbols with a modified subscript and with odd numbers as entries.

Definition 4.1. An odd Durfee symbol of $n$ is a two-rowed array with a subscript

$$
(\alpha, \beta)_{D}=\left(\begin{array}{cccc}
\alpha_{1} & \alpha_{2} & \cdots & \alpha_{s} \\
\beta_{1} & \beta_{2} & \cdots & \beta_{t}
\end{array}\right)_{D},
$$

where $\alpha_{i}$ and $\beta_{i}$ are all odd numbers, $2 D+1 \geq \alpha_{1} \geq \alpha_{2} \geq \cdots \geq \alpha_{s}>0$, $2 D+1 \geq \beta_{1} \geq \beta_{2} \geq \cdots \geq \beta_{t}>0$, and $n=\sum_{i=1}^{s} \alpha_{i}+\sum_{i=1}^{t} \beta_{i}+2 D^{2}+2 D+1$.

The odd rank of an odd Durfee symbol is defined as the number of parts of $\alpha$ minus the number of parts of $\beta$. Letting $\mathcal{D}_{1}^{0}(m ; n)$ denote the number of odd Durfee symbols of $n$ with odd rank $m$, we then have

Theorem 4.2. The generating function for $\mathcal{D}_{1}^{0}(m ; n)$ is given by

$$
\sum_{n=0}^{+\infty} \mathcal{D}_{1}^{0}(m ; n) q^{n}=\frac{1}{\left(q^{2} ; q^{2}\right)_{\infty}} \sum_{n=0}^{+\infty}(-1)^{n} q^{3 n^{2}+3 n+1+|m|(2 n+1)} .
$$


This result can easily follow by comparing the coefficients of $z^{m}$ in (4.3) given by Andrews [4, (8.4)-(8.5)]:

$$
\begin{aligned}
\sum_{n=1}^{+\infty} \sum_{m=-\infty}^{+\infty} \mathcal{D}_{1}^{0}(m ; n) z^{m} q^{n} & =\sum_{n \geq 0} \frac{q^{2 n(n+1)+1}}{\left(z q ; q^{2}\right)_{n+1}\left(z^{-1} q ; q^{2}\right)_{n+1}} \\
& =\frac{1}{\left(q^{2} ; q^{2}\right)_{\infty}} \sum_{n=-\infty}^{+\infty} \frac{(-1)^{n} q^{3 n^{2}+3 n+1}}{1-z q^{2 n+1}}
\end{aligned}
$$

where the first equality follows by a direct combinatorial argument, and the second equality is given by [20, p.66].

Andrews [4] also defined the $k$ th symmetrized odd rank moment by

$$
\eta_{k}^{0}(n)=\sum_{m=-\infty}^{+\infty}\left(\begin{array}{c}
m+\left\lfloor\frac{k-1}{2}\right\rfloor \\
k
\end{array}\right) \mathcal{D}_{1}^{0}(m ; n)
$$

and introduced $k$-marked odd Durfee symbols, whose definition is almost identical to that of $k$-marked Durfee symbols (Definition 1.4).

Definition 4.3. A $k$-marked odd Durfee symbol of $n$ is composed of $k$ pairs of partitions into odd parts with a subscript, which is defined as

$$
\eta^{0}=\left(\begin{array}{cccc}
\alpha^{k}, & \alpha^{k-1}, & \ldots, & \alpha^{1} \\
\beta^{k}, & \beta^{k-1}, & \ldots, & \beta^{1}
\end{array}\right)_{D}
$$

where $\alpha^{i}$ (resp. $\left.\beta^{i}\right)$ are all partitions with odd parts and $\sum_{i=1}^{k}\left(\left|\alpha^{i}\right|+\left|\beta^{i}\right|\right)+2 D^{2}+$ $2 D+1=n$. Furthermore, the partitions $\alpha^{i}$ and $\beta^{i}$ must satisfy almost the same conditions with $k$-marked Durfee symbols except for the third term in Definition 1.4, where for the $k$ th vector $\left(\alpha^{k}, \beta^{k}\right)$ of $k$-marked odd Durfee symbols, $\beta_{1}^{k}, \alpha_{1}^{k} \leq 2 D+1$.

Following a $k$-marked Durfee symbol, Andrews defined the $i$ th odd rank for a $k$-marked odd Durfee symbol. For a $k$-marked odd Durfee symbol $\eta^{0}$, we define $\rho_{i}\left(\eta^{0}\right)$, the $i$ th odd rank of $\eta^{0}$ by

$$
\rho_{i}\left(\eta^{0}\right)= \begin{cases}l\left(\alpha^{i}\right)-l\left(\beta^{i}\right)-1 & \text { for } 1 \leq i<k, \\ l\left(\alpha^{k}\right)-l\left(\beta^{k}\right) & \text { for } i=k .\end{cases}
$$

Let $\mathcal{D}_{k}^{0}\left(m_{1}, m_{2}, \ldots, m_{k} ; n\right)$ denote the number of $k$-marked odd Durfee symbols of $n$ with $i$ th odd rank equal to $m_{i}$ and let $\mathcal{D}_{k}^{0}(n)$ denote the number of $k$-marked odd Durfee symbols of $n$. Define $R_{k}^{0}\left(x_{1}, x_{2}, \ldots, x_{k} ; q\right)$ by

$$
R_{k}^{0}\left(x_{1}, x_{2}, \ldots, x_{k} ; q\right)=\sum_{m_{1}, \ldots, m_{k}=-\infty}^{+\infty} \sum_{n=0}^{+\infty} \mathcal{D}_{k}^{0}\left(m_{1}, m_{2}, \ldots, m_{k} ; n\right) x_{1}^{m_{1}} x_{2}^{m_{2}} \cdots x_{k}^{m_{k}} q^{n} .
$$

Andrews deduced the following four identities on $k$-marked odd Durfee symbols which are much similar to $k$-marked Durfee symbols.

Theorem 4.4 (Corollary 27, Andrews 4]).

$$
R_{k}^{0}\left(x_{1}, x_{2}, \ldots, x_{k} ; q\right)=\frac{1}{\left(q^{2} ; q^{2}\right)_{\infty}} \sum_{n=0}^{+\infty}(-1)^{n} q^{3 n^{2}+(2 k+1) n+k} \frac{1-q^{4 n+2}}{\prod_{j=1}^{k}\left(1-x_{j} q^{n}\right)\left(1-\frac{q^{n}}{x_{j}}\right)} .
$$


Theorem 4.5 (Corollary 28, Andrews [4). $\mathcal{D}_{k}^{0}\left(m_{1}, m_{2}, \ldots, m_{k} ; n\right)$ is symmetric in $m_{1}, m_{2}, \ldots, m_{k}$.

Theorem 4.6 (Corollary 29, Andrews [4]). For $k \geq 1$,

$$
\mathcal{D}_{k+1}^{0}(n)=\eta_{2 k}^{0}(n) \text {. }
$$

Theorem 4.7 (Theorem 25, Andrews [4]).

$$
R_{k}^{0}\left(x_{1}, x_{2}, \ldots, x_{k} ; q\right)=\sum_{i=1}^{k} \frac{R_{1}^{0}\left(x_{i} ; q\right)}{\prod_{\substack{j=1 \\ j \neq i}}^{k}\left(x_{i}-x_{j}\right)\left(1-\frac{1}{x_{i} x_{j}}\right)} .
$$

We now give a brief expository of how to prove these four conclusions combinatorially. First of all, it's straightforward to see that the bijection $\Phi$ in Theorem 2.1, the bijection $\Psi$ in Theorem 2.6 and the bijection $\Theta$ in Theorem 2.10 in Section 2 are valid for $k$-marked odd Durfee symbols. One then easily deduces the same result for $k$-marked odd Durfee symbols as Theorem 1.5 .

Theorem 4.8. For $k \geq 2$, we have

$$
\mathcal{D}_{k}^{0}\left(m_{1}, m_{2}, \ldots, m_{k} ; n\right)=\sum_{j=0}^{+\infty}\left(\begin{array}{c}
j+k-2 \\
k-2
\end{array}\right) \mathcal{D}_{1}^{0}\left(\sum_{i=1}^{k}\left|m_{i}\right|+2 j+k-1 ; n\right) .
$$

Thus, Theorems 4.5, 4.6, and 4.7 can be deduced from Theorem 4.8 by precisely the same progressions as Theorems 3.2, 3.3, and 3.4 in Section 3. To prove Theorem 4.4. it suffices to prove that the right hand side of (4.5) is the generating function for the sum on the right hand side of (4.8), which can be easily derived by Theorem 4.2, following the same progression as Theorem 3.1

\section{ACKNOWLEDGMENTS}

The author would like to thank Guoce Xin and Yue Zhou for helpful discussions, and she is grateful to George E. Andrews for valuable comments. This work was supported by the 973 Project, the PCSIRT Project and the Doctoral Program Fund of the Ministry of Education and the National Science Foundation of China.

\section{REFERENCES}

1. G. E. Andrews, Problems and prospects for basic hypergeometric functions, In: R. Askey, Theory and Application of Special Functions, Academic Press, New York, 1975, pp.191-224. MR.0399528 (53:3372)

2. G. E. Andrews, The Theory of Partitions, Addison-Wesley Publishing Co., 1976. MR0557013 $(58: 27738)$

3. G. E. Andrews, Plane partitions (III): The weak Macdonald conjecture, lnvent. Math. 53 (1979) 193-225. MR549398 (80j:05009)

4. G. E. Andrews, Partitions, Durfee symbols, and the Atkin-Garvan moments of ranks, Invent. Math. 169 (2007) 37-73. MR2308850(2008d:05013)

5. A. O. L. Atkin and P. Swinnerton-Dyer, Some properties of partitions, Proc. Lond. Math. Soc. III. Ser. 4 (1954) 84-106. MR0060535(15:685d)

6. A. O. L. Atkin and F. Garvan, Relations between the ranks and cranks of partitions, Ramanujan J. 7 (2003) 343-366. MR2035811 (2005e:11131)

7. A. Berkovich and F. G. Garvan, Some observations on Dyson's new symmetries of partitions, J. Combin. Theory Ser. A 100 (2002) 61-93. MR1932070 (2003g:11115)

8. C. Boulet and K. Kursungoz, Symmetry of k-marked Durfee symbols, Int. J. Number Theory, to appear. 
9. K. Bringmann, F. Garvan, and K. Mahlburg, Partition statistics and quasiharmonic Maass forms, Int. Math. Res. Not. (2009) Art. ID rnn124, 63-97. MR2471296 (2009j:11073)

10. K. Bringmann, On the explicit construction of higher deformations of partition statistics, Duke Math. J. 114 (2008) 195-233. MR2437679 (2009e:11203)

11. K. Bringmann, J. Lovejoy, and R. Osburn, Automorphic properties of generating functions for generalized rank moments and Durfee symbols, Int. Math. Res. Not. IMRN 2 (2010) 238-260. MR.2581041

12. F. J. Dyson, Some guesses in the theory of partitions, Eureka (Cambridge) 8 (1944) 10-15.

13. F. J. Dyson, A new symmetry of partitions, J. Combin. Theory Ser. A 7 (1969) 56-61. MR0238711 (39:75)

14. I. M. Gessel and G. Xin, A short proof of the Zeilberger-Bressoud q-Dyson theorem, Proc. Amer. Math. Soc. 134 (2006) 2179-2187. MR2213689 (2007e:05012)

15. W. J. Keith, Rank of Partitions and Degree Symbols, The Pennsylvania State University, 2007.

16. W. J. Keith, Distribution of the full rank in residue classes for odd moduli, Discrete Math. 309 (2009) 4960-4968. MR2548896

17. K. Kursungoz, Parity Considerations in Andrews-Gordon Identities and the $k$-Marked Durfee Symbols, The Pennsylvania State University, 2009.

18. G. Xin, A fast algorithm for MacMahon's partition analysis, Electron. J. Combin. 11 (2004) R58 20 pp. MR2097324 (2005h:05015)

19. G. Xin, A residue theorem for Malcev-Neumann series, Adv. Appl. Math. 35 (2005) 271-293. MR 2164920(2006m:16061)

20. G. N. Watson, The final problem, J. Lond. Math. Soc. 11 (1936) 55-80.

Center for Combinatorics, LPMC-TJKLC, Nankai University, Tianjin 300071, PeoPLE'S REPUBLIC OF CHINA

E-mail address: ji@nankai.edu.cn 